\title{
Optimal time for capillary blood glucose monitoring in patients with type 2 diabetes treated with insulin: a cross-sectional observation study
}

\begin{abstract}
Our objective was to determine the frequency and time of day when we detect the greatest number of hyperglycaemic and hypoglycaemic episodes in patients with type 2 diabetes mellitus (DM) on insulin. We also studied whether any clinical feature could be associated with more frequent hypoglycaemia or hyperglycaemia.

We conducted an observational study of 51 patients with type 2 diabetes mellitus who were on insulin and metabolically stable. We followed the patients for 8 weeks, performing two daily capillary blood glucose measurements before and 2hours after each of the three main meals in turn. A profile was also prepared from 6 capillary blood glucose measurements performed before and after meals every 4 days.

The likelihood of observing hyperglycaemia increased $48 \%$ before dinner (taking pre-breakfast capillary blood glucose as a reference). The likelihood of observing hypoglycaemia was 3.4 times greater in pre-lunch capillary blood glucose measurements and was more frequent in patients with lower BMI

In patients with type $2 \mathrm{DM}$ stabilised by insulin, performing pre-dinner capillary blood glucose measurements identifies hyperglycaemia more often than at other times of the day, while pre-lunch measurement identifies hypoglycaemia more often, the latter being more common in patients with lower BMI.
\end{abstract}

Keywords: type 1 diabetes mellitus, type 2 diabetes mellitus, insulin, capillary blood glucose
Volume I Issue 2 - 2014

\author{
Garcia Lopez JM,' Pazos Couselo M,' \\ Gonzalez Rodriguez M,' Gude Sampedro F', \\ Mayan Santos JM, ${ }^{3}$ Rodriguez J, ${ }^{4}$ Segade $\mathrm{S},{ }^{4}$ \\ Casanueva $\mathrm{FF}^{\mathrm{I}, 5,6}$ \\ 'Department of Endocrinology, The University Hospital, \\ University of Santiago de Compostela, Spain \\ 2Department of Clinical Epidemiology, The University Hospital, \\ University of Santiago de Compostela, Spain \\ ${ }^{3}$ Gerontology School of Nursing, University of Santiago de \\ Compostela, Spain \\ ${ }^{4}$ Clinical Biochemistry Laboratory, The University Hospital \\ University of Santiago de Compostela, Spain \\ DDepartment of Medicine, Santiago de Compostela University, \\ The University Hospital, Spain \\ ${ }^{6} \mathrm{CIBER}$ of Obesity Pathophysiology and Nutrition (СB06/03), \\ Carlos III Health Institute, Spain
}

Correspondence: JM Garcia Lopez, Department of Endocrinology, The University Hospital, Dr. R. Baltar sn 15702 , Santiago de Compostela, Spain, Tel 003498195 161I, Email jose.manuel.garcia.lopez@sergas.es

Received: May 02, 2014 | Published: June 19, 2014
Abbreviations: BMI, body mass index; DM, diabetes mellitus; SMBG, self monitoring of blood glucose; OAD, oral antidiabetic agents; JDS, japanese diabetes society; JSCC, japanese society for clinical chemistry; DCCT, diabetes control and complications trial; GLMM, generalised linear mixed models; OR, odds ratios; CI, confidence intervals

\section{Introduction}

The self monitoring of blood glucose (SMBG) provides real-time information that helps patients to manage their diabetes, especially patients on an insulin regime. The purpose of SMBG is to facilitate appropriate clinical interventions to achieve and maintain blood glucose within an acceptable range by detecting hyperglycaemia and hypoglycaemia, thus improving metabolic control and patient satisfaction.

The recommendations for SMBG are not uniform but differ among countries and patients. They depend on treatment intensity and metabolic state and they are often influenced by cost. International guidelines recommend that the frequency and timing of SMBG should be determined by the needs and goals of each patient. More frequent monitoring may be appropriate for poorly controlled patients; in contrast, stable patients who do not adjust insulin doses require less testing.

Although we provide patients with instructions on the number of measurements and times of day when they should perform self- monitoring, there is no well-defined pattern in such instructions ${ }^{2-4}$ and major discrepancies concerning the recommendations for metabolic self-monitoring are found in patients with type 2 diabetes receiving conventional insulin therapy. Usually, SMBG preprandial and postprandial are recommended but the timing and number of measurements vary highly and frequently are performed in an erratic way. Our hypothesis is that a structured SMBG program with readings at specific times may provide a thorough information of blood glucose patterns that allowed us to drive the right treatment. ${ }^{5,6}$

The aim of this study was to investigate the frequency and optimal time of SMBG in patients with type 2 diabetes on insulin and with stable glycaemic control. In addition, the clinical features associated with episodes of hypoglycaemia and/or hyperglycaemia was investigated.

\section{Materials and methods}

Observational study involving 51 patients ( 20 men and 31 women) who met the following criteria:

a) Older than 40years

b) Body mass index (BMI) $<40 \mathrm{~kg} / \mathrm{m}^{2}$

c) Type 2 diabetes mellitus (DM) of at least 1year duration

d) On insulin therapy for at least 3 months before recruitment

e) Ability to perform self-monitoring and 
f) Stable metabolic situation, defined as having no need to add new treatments or make changes in insulin dose $>10 \%$. $^{7}$ Oral antidiabetic agents (OAD) used: metformin(69\%), DPP-IV inhibitors $(16 \%)$, sulfonylureas $(10 \%)$ and glinides $(5 \%)$. The patients were recruited when they came to their routine check at the University Hospital of Santiago de Compostela, Spain. The recruitment period was from July 2012 to May 2013. The clinical characteristics of the participants are described in (Table 1).

Table I Clinical characteristics of patients

\begin{tabular}{ll}
\hline $\mathrm{N}$ & $5 \mathrm{I}$ \\
Men & $20(39 \%)$ \\
Women & $31(61 \%)$ \\
\hline Age(years) & $62 \pm 7$ \\
Duration of diabetes(years) & $1 \mathrm{I} \pm 7$ \\
Age range(years) & 49.77 \\
BMI(kg/m $\left.{ }^{2}\right)$ & $31.8 \pm 3.9$ \\
Treatment & \\
Insulin alone & 18 \\
Insulin+oral monotherapy & $21(64 \%)$ \\
Insulin+OAD Combination & $12(36 \%)$ \\
Insulin** & \\
A & $16(31 \%)$ \\
B & $15(30 \%)$ \\
C & $20(39 \%)$ \\
\hline
\end{tabular}

*Treatment with oral antidiabetic agents; **A, Basal insulin; B, Mixtures of standard insulin (30/70,50/50); C, Basal insulin+3-rapid insulin

The initial consultation occurred after obtaining informed consent and included a complete clinical evaluation of the patient, who was instructed on SMBG using BG Star ${ }^{\circledR}$ (Sanofi-Aventis). The patients were instructed to record daily blood glucose measurements and to maintain their usual diet.
During a period of 8 weeks, the patients underwent structured preand post-prandial blood glucose monitoring at the three main meals, alternating throughout the week following the scheme in (Table 2) (Day 1: pre-breakfast and 2hours post-breakfast, Day 2: pre-lunch and 2hours post-lunch, Day 3: pre-dinner and 2hours post-dinner and Day 4: pre- and post-prandial 6-point profile). Plasma HbA1c was determined at the end of week 8 .

The HbAlc was determined by high-performance liquid chromatography in a Menarini Diagnostics HA-8140 analyser. All $\mathrm{HbA1c}$ values were converted from the Japanese Diabetes Society (JDS)/Japanese Society for Clinical Chemistry (JSCC)-referenced values to Diabetes Control and Complications Trial (DCCT)-aligned units using the following equation: A1CNGSP $=0.985$ A1CJDS/ JSCC $+0.46 \%$, where NGSP is the National Glycohaemoglobin Standardisation Program. ${ }^{8}$

Hyperglycaemia was defined as pre-prandial capillary blood glucose $>7.22 \mathrm{mmol} / 1$ and post-prandial capillary blood glucose $>10 \mathrm{mmol} / 1 .{ }^{9}$ Hypoglycaemia was defined as capillary blood glucose values $<3.89 \mathrm{mmol} / 1$. Out of range readings were defined as preprandial or postprandial glucose values beyond these limits.

\section{Statistical analyses}

Descriptive statistical analyses were used to analyse and describe the clinical characteristics and patient demographics. The means $\pm \mathrm{SD}$ are reported for continuous variables.

Generalised Linear Mixed Models (GLMM), with the patient and timing as random effects, were used to investigate the clinical characteristics associated with episodes of hypoglycaemia or hyperglycaemia. In both models the variables age, gender, BMI, time from diagnosis, timing, type of treatment and $\mathrm{HbAlc}$ levels were included as fixed effects. A binomial distribution and its canonical link function (log it) were implemented to model the presence/ absence of these episodes (hypoglycaemia/hyperglycaemia), which allowed us to express the results in terms of odds ratios (OR) and their $95 \%$ confidence intervals (CI). The effect is statistically significant at the $5 \%$ level if the CI does not include 1 . Statistical analyses were performed using the lme4 R package. ${ }^{10}$

Table 2 Capillary blood glucose schedule

\begin{tabular}{|c|c|c|c|c|c|c|}
\hline Day & Pre-breakfast & Post-breakfast & Pre-lunch & Post-lunch & Pre-dinner & Post-dinner \\
\hline I & $x$ & $x$ & & & & \\
\hline 2 & & & $x$ & $x$ & & \\
\hline 3 & & & & & $x$ & $x$ \\
\hline 4 & $x$ & $x$ & $x$ & $x$ & $x$ & $x$ \\
\hline 5 & $x$ & $x$ & & & & \\
\hline 6 & & & $x$ & $x$ & & \\
\hline 7 & & & & & $x$ & $x$ \\
\hline
\end{tabular}

\section{Results}

Of the 65 patients recruited for the study, 51 completed all 8 weeks of recording capillary blood glucose measurements; 5 did not they complete the study; and 9 were excluded for not meeting the inclusion criteria. Of the 144 capillary blood glucose measurements scheduled for every patient within the 8 weeks, $93 \%$ of them were performed.
(Figure 1) shows the capillary blood glucose measurements of all participants according to the time of measurement and $\mathrm{HbA} 1 \mathrm{c}$ levels.

A direct relationship can be observed between the capillary blood glucose and $\mathrm{HbA1c}$ levels (Figure 1). The HbAlc levels at baseline and at the end of the study were $60 \pm 10 \mathrm{mmol} / \mathrm{mol}(7.6 \pm 1.3 \%)$ and $59 \pm 10 \mathrm{mmol} / \mathrm{mol}(7.5 \pm 1.3 \%)$, respectively. 


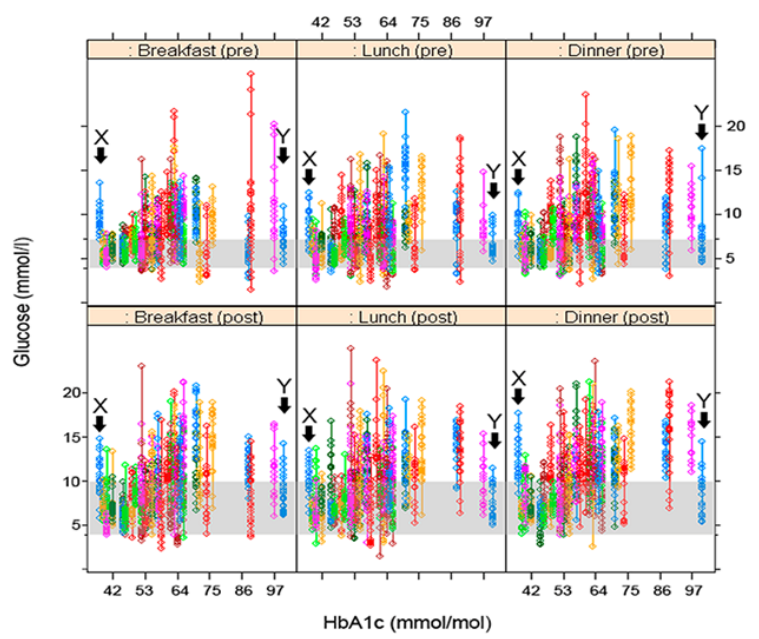

Figure I Capillary blood glucose pre and post prandial Vs HbAlc values during the 8-weeks period. Each line corresponds to a patient and his capillary blood glucose values. $X$ and $Y$ are patients with similar capillary blood glucose and very different $\mathrm{HbAlc}$ values.
(Table 3) lists the mean pre- and post-prandial capillary blood glucose measurements along with the hypoglycaemias and hyperglycaemias and their percentage. The lowest blood glucose levels appear before meals and are highest after meals, with increases over the day.

\section{Hyperglycaemia}

All patients had at least one episode of pre-prandial hyperglycaemia and most (92\%) also had at least 1 episode of postprandial hyperglycaemia. (Table 4) shows the results of multivariate analysis. Of the clinical variables analysed (age, sex, BMI, HbA1c, time from diagnosis and type of treatment), only elevated HbA1c levels were significantly associated with the occurrence of episodes of hyperglycaemia $(\mathrm{p}<0.001)$.

Considering pre-breakfast capillary blood glucose levels as the reference, the likelihood of hyperglycaemia increases by $48 \%$ in predinner blood glucose measurements $(p<0.001)$ and decreases in the post-breakfast $(p<0.001)$ and post-lunch $(p<0.001)$ measurements. At other times of the day, no statistical significance was obtained. By measuring the pre-dinner blood glucose, we captured $20.6 \%$ of the hyperglycaemic episodes.

Table 3 Mean glucose levels and proportion of hypoglycemic, hyperglycemic and out-of-range readings captured at different testing times

\begin{tabular}{llllll}
\hline & Valid Reading (\%) & $\begin{array}{l}\text { Mean } \pm \text { SD } \\
(\mathbf{m m o l} / \mathbf{l})\end{array}$ & $\begin{array}{l}\text { Hypoglycemic } \\
\text { readings (\%) }\end{array}$ & $\begin{array}{l}\text { Hyperglycemic } \\
\text { readings (\%) }\end{array}$ & $\begin{array}{l}\text { Out-of-range } \\
\text { readings (\%) }\end{array}$ \\
\hline Total & $6648(93.2)$ & $8.8 \pm 3.3$ & $135(100)$ & $3209(100)$ & $3344(100)$ \\
Pre-breakfast & $1168(95.4)$ & $7.7 \pm 2.5$ & $26(19.2)$ & $600(18.7)$ & $626(18.7)$ \\
Post-breakfast & $1135(92.7)$ & $9.2 \pm 3.5$ & $20(14.8)$ & $383(11.9)$ & $403(12.0)$ \\
Pre-lunch & $1154(94.3)$ & $7.8 \pm 2.9$ & $43(31.9)$ & $573(17.8)$ & $616(18.4)$ \\
Post-lunch & $1128(92.1)$ & $9.5 \pm 3.3$ & $19(14.1)$ & $430(13.4)$ & $449(13.4)$ \\
Pre-dinner & $1146(93.6)$ & $8.4 \pm 3.2$ & $20(14.8)$ & $662(20.6)$ & $682(20.4)$ \\
Post-dinner & $1117(91.2)$ & $10.4 \pm 3.6$ & $7(5.2)$ & $561(17.5)$ & $568(17.0)$ \\
\hline
\end{tabular}

Table 4 Timings and clinical variables associated with hyperglycemia and hypoglycemia

\begin{tabular}{|c|c|c|c|c|}
\hline & Hyperglycemia & & Hypoglycemia & \\
\hline & OR(95\% Cl) & p-value & OR(95\% Cl) & p-value \\
\hline Age(years) & $1.02(0.97,1.08)$ & 0.403 & $0.93(0.85,1.02)$ & 0.143 \\
\hline Sex(male/female) & $1.09(0.39,1.65)$ & 0.54 & $0.67(0.20,2.2 \mathrm{I})$ & 0.511 \\
\hline $\operatorname{BMI}\left(\mathrm{kg} / \mathrm{m}^{2}\right)$ & $0.99(0.91,1.09)$ & 0.91 & $0.77(0.66,0.90)$ & 0.001 \\
\hline Time of diagnosis & $1.01(0.96,1.06)$ & 0.733 & $1.02(0.94, \mathrm{I} . \mathrm{II})$ & 0.563 \\
\hline \multicolumn{5}{|l|}{ Treatment } \\
\hline A(basal) & Ref & & Ref & \\
\hline B(basal + rapid) & $2.30(0.90,5.90)$ & 0.083 & $0.97(0.26,3.63)$ & 0.967 \\
\hline$C$ (basal + 3 rapid) & I. $10(0.50,2.4 \mathrm{I})$ & 0.813 & $1.03(0.28,3.88)$ & 0.958 \\
\hline $\mathrm{HbAlc}, \%$ & I.82(I.37, 2.4I) & 0 & $1.03(0.63,1.69)$ & 0.902 \\
\hline \multicolumn{5}{|l|}{ Capillary glycemia } \\
\hline Time & Ref & & Ref & \\
\hline Pre-breakfast & $0.38(0.3 \mathrm{I}, 0.47)$ & 0 & $0.44(0.16,1.17)$ & 0.101 \\
\hline Post-breakfast & $0.91(0.75,1.10)$ & 0.338 & $3.40(1.28,9.00)$ & 0.013 \\
\hline
\end{tabular}

Citation: Garcia Lopez JM, Pazos Couselo M, Gonzalez Rodriguez M, et al. Optimal time for capillary blood glucose monitoring in patients with type 2 diabetes treated with insulin: a cross-sectional observation study.J Diabetes Metab Disord Control. 20 I4; I (2):4I-45. DOI: I0.15406/jdmdc.20I4.01.000 I0 
Table Continued...

\begin{tabular}{lllll}
\hline & Hyperglycemia & & Hypoglycemia & \\
\hline & OR(95\% Cl) & p-value & OR(95\% Cl) & p-value \\
\hline Pre-lunch & $0.52(0.43,0.63)$ & 0 & $1.39(0.48,4.05)$ & 0.54 \\
Post-lunch & $1.48(1.22,1.80)$ & 0 & $1.20(0.44,3.32)$ & 0.722 \\
Pre-dinner & $1.03(0.84,1.25)$ & 0.803 & $0.26(0.06,1.11)$ & 0.07 \\
Post-dinner & & & &
\end{tabular}

OR, odd ratio; $95 \% \mathrm{Cl}$, 95\% confidence interval; BMI, body mass index; HbA Ic, glycated hemoglobin; treatment A; Ref, reference category

\section{Hypoglycaemia}

There were 135 hypoglycaemic values, representing $1.97 \%$ of the capillary blood glucose measurements performed. Thirty-seven per cent (19 patients) had at least one hypoglycaemic episode. In the univariable analysis, episodes of hipoglucemia were more frequent in patients treated with basal insulin $(34.6 \%)$ and in patients treated with mixtures of standard insulin $(34.6 \%)$ than in patients treated with basal insulin added to 3 rapid insulin $(30.8 \%)$, but these differences did not reach statistical significance. However after adjusting for HbA1c, BMI, age, sex and time from diagnosis, insulin treatment was not associated with hypoglycaemia events. Adding oral antidiabetic drugs was not associated with hypoglycaemia events.

Regarding the clinical variables studied, only BMI was significantly associated with hypoglycaemia $(\mathrm{p}<0.001)$, with a higher frequency of episodes in individuals with lower BMI. Considering the prebreakfast capillary blood glucose levels as reference, the likelihood of hypoglycaemia is 3.4times greater in the pre-lunch capillary blood glucose measurements $(p<0.001)$. By measuring pre-lunch blood glucose, we captured $31.9 \%$ of the hypoglycaemic episodes.

\section{Discussion}

The level of compliance with the performance of capillary blood glucose measurements in our group (93\%) was similar to other studies. ${ }^{11}$ Despite the large number of glucose measurements performed during these 8 weeks, the mean HbAlc levels decreased slightly from $60 \mathrm{mmol} / \mathrm{mol}(7.6 \%)$ to $59 \mathrm{mmol} / \mathrm{mol}(7.5 \%)$, indicating that the patients were in a stable metabolic state. It is also expected that patients with approximately normal $\mathrm{HbAlc}$ values would not present great changes in their SMBG values. ${ }^{6,12-14}$

The most important result of this study in patients with stable insulin-treated type 2 diabetes mellitus is that the times of day when hyperglycaemia or hypoglycaemia occurs more frequently can be identified and associated with the clinical characteristics of the patients. Specifically, in this group of patients, hyperglycaemia is more frequent in subjects with elevated HbAlc levels and in pre-dinner capillary measurements, while hypoglycaemia is more common in subjects with lower BMI and pre-lunch measurements. The highest proportion of hyperglycemic readings was found at predinner and the highest proportion of hypoglycemic readings happened prelunch. Others authors ${ }^{7}$ concluded also that testing at prelunch/predinner times yielded the highest proportion of hypoglycemic and hyperglycemic events, respectively. The limitations of this study are that the patients included were only males and postprandial glucose measurements were not done.

Our results are more generalizables because we included female and our patients also performed postprandial glucose measurements giving a more complete glucose daily profile. Many studies have shown that HbAlc is an indicator of mean blood glucose levels. Therefore, it is expected that patients with higher HbAlc levels would have higher blood glucose levels and that hyperglycaemia would be associated with higher $\mathrm{HbAl} \mathrm{c}$ levels. $\mathrm{HbA} 1 \mathrm{c}$ is an indicator of overall glycaemic control, but it does not report glycaemic fluctuations and does not provide information about hypoglycemic episodes. Capillary blood glucose allows us to evaluate glycaemic variability apart from identifying hyperglycaemia and hypoglycaemia SMBG enables us a day to day better management of hypoglycemic and hyperglycemic events.

Hypoglycaemia and the fear of hypoglycaemia has been a limiting factor for many patients when attempting to achieve blood glucose levels within the normal range. Other authors found in a study design similar to ours ${ }^{7}$ that $2 \%$ of patients experienced hypoglycaemic episodes. In this study, the largest proportion of hypoglycaemic episodes occurred before lunch. There are a number of possible causes of hypoglycaemia, but the most common are related to diabetes medication, the amount of food eaten, or the level of exercise performed by the patient. Low BMI was also identified as a predictor of hypoglycaemia. Other studies found that low BMI was associated with hypoglycaemia in patients with type 2 diabetes on insulin. ${ }^{7,15,16}$ This result can be explained by the fact that patients with lower average BMI may show increased sensitivity to insulin, which thus represents a greater risk for them. Neither the type of treatment (insulin alone, insulin added to oral agents, insulin mixtures or prandial insulin added to basal insulin) age, sex or duration of diabetes were associated with more frequency of hyperglycemic or hypoglycemic readings.

SMBG is a widely accepted method for improving glycaemic control in patients with type 2 diabetes on insulin., ${ }^{1,2,6,7,12-14,17-19}$ The improvements in glycaemic control with SMBG have also demonstrated profitability, producing long-term incremental costeffectiveness ratios. ${ }^{12,17,20}$ Choosing an effective strategy for capillary blood glucose monitoring also depends on the capture of out-of-range readings. Our findings suggest that strategies including predinner and prelunch readings can capture a significative proportion of out of range glucose values.

Observational studies of SMBG are susceptible to biases that have already been well described. The causal interpretation of these results is limited by the lack of randomisation. However, randomised controlled trials also have limitations for the study of SMBG. SMBG blind studies are not possible and the current SMBG practice guidelines make it unethical to include a group without exposure. Similarly, in our study, we did not evaluate whether the subjects with hypoglycaemic measurements were symptomatic.

This study was conducted within a well-defined framework of patients; all subjects were patients with stable insulin-treated type 2 diabetes mellitus. On the other hand, lifestyles (dietary habits, 
exercise) and patterns of insulin therapy can vary substantially among countries. Therefore, these results cannot be generalised to other patient groups or other situations.

\section{Conclusion}

In patients with stable insulin-treated type 2 diabetes mellitus, the times of day at which the occurrence of hyperglycaemia or hypoglycaemia is more frequent can be identified. The onset of hyperglycaemia is more frequent in subjects with elevated $\mathrm{HbA1c}$ levels and in pre-dinner measurements. The occurrence of hypoglycaemia is more common in subjects with lower BMI and in pre-lunch measurements.

\section{Acknowledgements}

This study was supported by grants from the Carlos III Health Institute, Spain (PI11/02219) and Sanofi Aventis. The authors are solely responsible for the design and conduct of this study; all study analyses, the drafting and editing of the manuscript and its final contents.

\section{Novelty statement}

The self monitoring of capillary blood glucose plays an important role in the control of diabetes mellitus. The ideal number of measurements to be performed and time of day have not been well established. Our study shows that predinner and prelunch capillary blood glucose captures a higher proportion of hyperglycemia and hypoglycemia, respectively. Patients with lower body mass index (BMI) have a higher frequency of hypoglycemic episodes.

This information can help to optimise the use of capillary blood glucose measurements in the management of patients with type 2diabetes mellitus stabilised by insulin.

\section{Conflict of interest}

Author declares that there is no conflict of interest.

\section{References}

1. Duran A, Martin P, Runkle I, et al. Benefits of self-monitoring blood glucose in the management of new-onset Type 2 diabetes mellitus: The $\mathrm{S}$ Carlos Study, a prospective randomized clinic-based interventional study with parallel groups. J Diabetes. 2010;2(3):203-211.

2. American Diabetes Association. Standards of medical care in diabetes-2013. Diabetes Care. 2013;36:S11-S66.

3. Allemann S, Houriet C, Diem P, et al. Self-monitoring of blood glucose in non-insulin treated patients with type 2 diabetes: a systematic review and meta-analysis. Curr Med Res Opin. 2009;25(12):2903-2913.

4. http://www.abcdiabetes.co.uk/abc-for-healthcare

5. Harris MI. Frequency of blood glucose monitoring in relation to glycemic control in patients with type 2 diabetes. Diabetes Care. 2001;24(6):979-982.
6. Murata GH, Shah JH, Hoffman RM, et al. Intensified blood glucose monitoring improves glycemic control in stable, insulin-treated veterans with type 2 diabetes: the Diabetes Outcomes in Veterans Study (DOVES). Diabetes Care. 2003;26(6):1759-1763.

7. Hoffman RM, Shah JH, Wendel CS, et al. Diabetes Outcome in Veterans Study. Evaluating once- and twice-daily self-monitored blood glucose testing strategies for stable insulin-treated patients with type 2 diabetes: the diabetes outcomes in veterans study. Diabetes Care. 2002;25(10):1744-1748.

8. Hoelzel W, Weykamp C, Jeppsson JO, et al. IFCC reference system for measurement of hemoglobin A1c in human blood and the national standardization schemes in the United States, Japan, and Sweden: a method-comparison study. Clin Chem. 2004;50(1):166-174.

9. Oliver S, Hasan A, Tadej B, et al. Addressing schemes of self-monitoring of blood glucose in type 2 diabetes: a European perspective and expert recommendation. Diabetes Technol Ther. 2011;13(9):959-965.

10. http://cran.r-project.org/web/packages/lme4/index.html

11. Evans JM, Newton RW, Ruta DA, et al. Frequency of blood glucose monitoring in relation to glycaemic control: observational study with diabetes database. BMJ. 1999;319(7202):83-86.

12. Karter AJ, Parker MM, Moffet HH, et al. Longitudinal study of new and prevalent use of self-monitoring of blood glucose. Diabetes Care. 2006;29(8):1757-1763.

13. Martin S, Schneider B, Heinemann L, et al. Self-monitoring of blood glucose in type 2 diabetes and long-term outcome: an epidemiological cohort study. Diabetologia. 2006;49(2):271-278.

14. O'Kane MJ, Bunting B, Copeland M, et al. Efficacy of self-monitoring of blood glucose in patients with newly diagnosed type 2 diabetes (ESMON study): randomised controlled trial. BMJ. 2008;336:1174-1177.

15. Seufert J, Brath H, Pscherer S, et al. Composite efficacy parameters and predictors of hypoglycaemia in basal-plus insulin therapy-a combined analysis of 713 type 2 diabetic patients. Diabetes Obes Metab. 2014;16(3):248-254.

16. Samann A, Lehmann T, Heller T, et al. A retrospective study on the incidence and risk factors of severe hypoglycemia in primary care. Fam Pract. 2013;30(3):290-293.

17. Tunis SL, Willis WD, Foos V. Self-monitoring of blood glucose (SMBG) in patients with type 2 diabetes on oral anti-diabetes drugs: costeffectiveness in France, Germany, Italy, and Spain. Curr Med Res Opin. 2010;26(1):163-175.

18. Sarwat S, Lag LL, Carey A, et al. The relationship between selfmonitored blood glucose values and glycated haemoglobin in insulintreated patients with Type 2 diabetes. Diabet Med. 2010;27(5):589-592.

19. Schutt M, Kern W, Krause U, et al. Is the frequency of self-monitoring of blood glucose related to long-term metabolic control? Multicenter analysis including 24,500 patients from 191 centers in Germany and Austria. Exp Clin Endocrinol Diabetes. 2006;114(7):384-388.

20. Palmer AJ, Dinneen S, Gavin JR, et al. Cost-utility analysis in a UK setting of self-monitoring of blood glucose in patients with type 2 diabetes. Curr Med Res Opin. 2006;22(5):861-72. 